\title{
Escalas de Práticas Parentais (EPP): Avaliando Dimensões de Práticas Parentais em Relação a Adolescentes
}

\author{
Parental Practices Scales (PPS): Evaluating Dimensions of \\ Parental Practices Towards Adolescents \\ Marco Antônio Pereira Teixeira*, Adriano Machado Oliveira, Shana Hastenpflug Wottrich \\ Universidade Federal de Santa Maria, Santa Maria, Brasil
}

\begin{abstract}
Resumo
O objetivo deste estudo foi elaborar um instrumento de auto-relato para avaliar dimensões de práticas educativas parentais em relação a adolescentes. Participaram do estudo 409 adolescentes $(57,9 \%$, com média de idade de 16,52 anos), dos quais 298 forneceram protocolos completos. Foram gerados inicialmente 62 itens para avaliar diversas dimensões de práticas parentais. A estrutura subjacente ao conjunto dos itens foi avaliada através de análises de componentes principais. Sucessivas análises permitiram chegar a uma solução interpretável de 6 componentes para um conjunto de 27 itens, onde cada item apresentou carga superior a 0,35 em apenas um componente. Os componentes identificados foram (e respectivos alpha de Cronbach das escalas correspondentes): controle punitivo $(0,73)$, supervisão do comportamento $(0,77)$, cobrança de responsabilidade $(0,70)$, intrusividade $(0,67)$, apoio emocional $(0,89)$ e incentivo à autonomia $(0,76)$. Os resultados mostram, portanto, que é possível distinguir empiricamente dimensões de práticas educativas parentais mais específicas que as dimensões de responsividade e exigência tipicamente mencionadas na literatura. Além disso, as escalas mostraram padrões de correlações diferenciados com alguns indicadores de desenvolvimento psicossocial na adolescência, sugerindo que seus efeitos sobre o desenvolvimento também são distintos.

Palavras-chave: Práticas parentais; estilos parentais; adolescentes.
\end{abstract}

\begin{abstract}
The aim of this study was to elaborate a self-report instrument to evaluate dimensions of parental educational practices concerning adolescents. The sample was composed of 409 adolescents $(57,9 \%$ women, with an average age of 16,52 years old), from which 298 provided complete protocols. Initially, 62 items were generated to evaluate different dimensions of parental practices. The underlying structure present in the set of items was evaluated using principal components analysis. Repeated analysis allowed an interpretable solution of 6 components for a set of 27 items, considering items with factor loadings greater than 0.35 in just one component. The identified components are listed below, together with the respective Cronbach alpha value of the correspondent scales: punitive control (0.73), behavioral supervision (0.77), responsibility demandingness (0.70), intrusiveness (0.67), emotional support (0.89) and autonomy granting (0.76). Therefore, the results show that it is possible to empirically distinguish more specific parental educational practices than the responsiveness and demandingness dimensions that are tipically mentioned in the literature. Moreover, the scales show different correlational patterns when some psychosocial development indicators are considered, suggesting that the effects over the development are also distinct.

Keywords: Parental practices; parental styles; adolescents.
\end{abstract}

O modo como os pais educam seus filhos adolescentes, assim como os efeitos que as práticas educativas parentais produzem no desenvolvimento psicossocial dos jovens, tem sido objeto de atenção de diversos trabalhos ao longo dos últimos anos (por exemplo, Bardagi, 2002; Juang \& Silbereisen, 1999, 2002; Laible \& Carlo, 2004; Lamborn, Mounts, Steinberg \& Dornbusch, 1991; Meesters \& Muris, 2004; Pacheco, Teixeira \& Gomes, 1999; Reppold, 2001; Shucksmith, Hendry \& Glendinning, 1995). Em seu conjunto, os estudos sugerem que as práticas parentais de fato

\footnotetext{
* Endereço para correspondência: Rua Ramiro Barcelos, 2600, sala 117, CEP 90035-003 - Porto Alegre, RS. E-mail: mapteixeira@yahoo.com.br

Os autores agradecem as sugestões dadas por dois revisores anônimos deste artigo.
}

estão associadas a diversos indicadores de desenvolvimento psicológico e comportamental na adolescência, tais como auto-estima, depressão, ansiedade, desempenho acadêmico, competência interpessoal, comportamentos agressivos, entre outros.

As práticas educativas parentais podem ser entendidas como conjuntos de comportamentos singulares emitidos pelos pais no processo de educação ou socialização dos filhos que levam a um resultado comum, resultado este que seria um objetivo ou meta dos pais com a socialização (Darling \& Steinberg, 1993). Um exemplo seria o esforço parental para que um filho desenvolva um senso de autonomia. Para isso, um pai ou uma mãe pode incentivar o filho a ter suas próprias idéias, estimulá-lo a dar o máximo de si ou deixá-lo aprender algumas coisas por experiência própria. 
Nem sempre, contudo, este resultado é deliberadamente planejado pelos pais; o modo de agir em relação aos filhos pode ser muitas vezes guiado por valores sobre os quais os pais podem não refletir ou mesmo não se dar conta.

Muitos dos estudos que tratam do impacto das práticas educativas parentais sobre o desenvolvimento dos filhos adotam uma abordagem tipológica, comumente denominada de estilos parentais (Maccoby \& Martin, 1983; Steinberg, 2001). Ou seja, eles partem de dimensões mais ou menos amplas de práticas parentais para então definir "tipos" ou estilos parentais, dependendo do padrão do comportamento parental em cada uma das dimensões consideradas. Os estilos parentais, portanto, podem ser entendidos como o clima emocional, ou contexto, dentro do qual práticas parentais específicas são implementadas (Darling \& Steinberg, 1993).

Duas dimensões principais de práticas educativas parentais têm sido usualmente descritas na literatura (Darling \& Steinberg, 1993; Maccoby \& Martin, 1983): uma relacionada com atitudes coercitivas por parte dos pais (como punições e proibições) e outra associada a comportamentos mais afetivos (como dar carinho, mostrar-se orgulhoso ou desapontado). Essas dimensões têm sido denominadas, em alguns estudos, de exigência (demandingness) e responsividade (responsiveness). A exigência engloba atitudes de controle do comportamento dos filhos, através do estabelecimento de regras e de limites, ao passo que a responsividade está associada a comportamentos de apoio emocional, comunicação bi-direcional entre pais e filhos, e de estímulo à autonomia dos jovens. A combinação dessas duas grandes dimensões tem sido utilizada para caracterizar quatro estilos parentais amplos: autoritário, autoritativo, indulgente e negligente (Maccoby \& Martin, 1983), compondo um modelo de estilos cujos efeitos sobre o desenvolvimento de adolescentes tem sido amplamente demonstrado por diversas pesquisas (Steinberg, 2001).

Pode-se questionar, contudo, até que ponto o agrupamento de práticas educativas parentais dentro das duas grandes dimensões de exigência e responsividade pode estar mascarando efeitos mais específicos das práticas, ou mesmo não contemplando certos aspectos de práticas parentais que podem ocorrer no contexto da interação dos pais com os filhos adolescentes. Por exemplo, em um estudo que verificou as propriedades psicométricas de duas escalas para avaliar exigência e responsividade com adolescentes brasileiros, Costa, Teixeira e Gomes (2000) identificaram três fatores através de análise fatorial, ainda que a previsão inicial (conforme o estudo original norte-americano de Lamborn et al., 1991) fosse de apenas dois fatores (houve um desdobramento da exigência em duas subdimensões: uma de controle parental explícito e outra de controle implícito). De modo similar, Weber, Prado, Viezzer e Brandenburg (2004), usando o mesmo instrumento, também identificaram as duas dimensões em exigência, e outras duas em responsividade (uma relacionada às interações afetivas pais-filhos e outra relacionada a ajudas e incentivos). Estes resultados sugerem que podem haver outras dimensões empiricamente distintas (ainda que relacionadas a exigência e responsividade), mas estas podem não estar sendo identificadas em função dos instrumentos utilizados.

Além disso, outras dimensões de práticas parentais também são referidas na literatura. Entre estas, podem ser citadas a intrusividade (falta de respeito, por parte dos pais, em relação à privacidade e intimidade dos adolescentes), a confiança, o envolvimento (apoio emocional), a monitoração (que inclui o quanto os pais sabem a respeito dos filhos), o controle psicológico (métodos de controle encobertos, como indução de culpa e pressão excessiva), a sensibilidade dos pais para com as demandas do adolescente, a previsibilidade ou consistência do comportamento parental, o nível de envolvimento dos pais com tarefas escolares, a rejeição parental, a superproteção, a tolerância, o uso de punição e o conflito (Claes, Lacourse, Bouchard \& Perucchini, 2003; Costa, Teixeira \& Gomes, 1998; Juang \& Silbereisen, 1999; Meesters \& Muris, 2004).

A fim de que possam ser desenvolvidas pesquisas acerca das influências das práticas educativas parentais em relação a adolescentes em nossa cultura, é necessário que existam instrumentos que permitam uma avaliação consistente das dimensões teórica e empiricamente relevantes das práticas parentais. Contudo, poucos estudos brasileiros publicados relacionados à construção ou adaptação de instrumentos sobre práticas ou estilos parentais foram localizados. Um deles, o Inventário de Práticas Parentais (Benetti \& Balbinotti, 2003) foi desenvolvido para ser respondido pelos pais a respeito de suas práticas educativas com os filhos na faixa de 6 a 10 anos. Já os instrumentos propostos por Gomide (2003), Costa, Teixeira e Gomes (2000) e Teixeira, Bardagi e Gomes (2004) privilegiam a percepção dos próprios adolescentes sobre as práticas de seus pais. O Inventário de Estilo Parental (Gomide, 2003) avalia oito dimensões de práticas parentais mais diretamente relacionadas ao desenvolvimento ou inibição de comportamentos antisociais, denominadas de monitoria positiva, comportamento moral, negligência, abuso físico e psicológico, disciplina relaxada, punição inconsistente e monitoria negativa. Embora inclua as dimensões de monitoria positiva e comportamento moral, consideradas práticas que desenvolvem o comportamento pró-social, este inventário não especifica outras possíveis dimensões que podem concorrer para um desenvolvimento psicossocial positivo, como sugerem os estudos que têm investigado o amplo construto denominado de responsividade.

Por sua vez, o trabalho de Costa, Teixeira e Gomes (2000) buscou traduzir e adaptar ao português o instrumento utilizado por Lamborn et al. (1991), originalmente criado em língua inglesa para medir as dimensões de responsividade e exigência. Estas escalas foram posteriormente revisadas, tendo-se ampliado o número de itens e modificado o sistema de respostas (Teixeira, Bardagi \& Gomes, 2004). Contudo, na revisão mencionada não foram identificadas novas dimensões ou subdimensões "internas" às dimensões de responsividade e exigência propostas originalmente. 
Nesta pesquisa propôs-se a realização de uma nova revisão das escalas propostas por Teixeira, Bardagi e Gomes (2004), ampliando o número de itens de forma a contemplar mais experiências cotidianas vividas por adolescentes brasileiros no que diz respeito às práticas educativas de seus pais, com o intuito de verificar se é possível, empiricamente, distinguir outras dimensões de práticas que não apenas a responsividade e a exigência (ou subdimensões dentro destas).

Em síntese, portanto, os objetivos da presente pesquisa foram: (a) construir um instrumento de auto-relato para avaliar dimensões de práticas educativas parentais em relação a adolescentes (a ser respondido pelos próprios jovens); (b) explorar, através de análise de componentes principais, a estrutura subjacente ao conjunto de itens criados; (c) avaliar a fidedignidade das escalas obtidas; e (d) correlacionar as escalas obtidas com outras variáveis psicológicas relevantes (auto-estima, auto-eficácia, abertura emocional e comportamento anti-social), a fim de verificar se os resultados são coerentes com indicações da literatura.

\section{Método}

\section{Desenvolvimento do Instrumento}

\section{Estudo preliminar e elaboração dos itens do instrumento.}

Os itens do instrumento foram elaborados a partir de três fontes: os instrumentos já existentes sobre dimensões de responsividade e exigência em português (Costa, Teixeira \& Gomes, 2000; Teix eira, Bardagi \& Gomes, 2004), as indicações da literatura sobre práticas parentais e sugestões trazidas por adolescentes em um estudo preliminar. O estudo preliminar consistiu na aplicação dos itens de um instrumento já existente (Teixeira, Bardagi \& Gomes, 2004) a uma amostra de 32 adolescentes, em situação de sala de aula, para que discutissem a clareza de seus conteúdos e sugerissem outras práticas educativas efetuadas por seus pais em seus cotidianos, a fim de enriquecer o questionário. Não houve sugestões de novas práticas propriamente ditas, mas os adolescentes ressaltaram um aspecto importante na relação estabelecida com seus pais: a confiança. Segundo os adolescentes, quando os pais confiam neles não há necessidade de haver um controle mais ostensivo do comportamento. Decidiu-se então criar itens para avaliar a dimensão "confiança" no instrumento. Quanto à clareza e pertinência dos itens, os adolescentes os julgaram adequados.

Assim, foi elaborada uma versão inicial do instrumento com 62 itens, distribuídos em 12 dimensões de práticas: controle punitivo (refere-se a atitudes e comportamentos dos pais que demonstram o exercício da autoridade parental através de práticas punitivas e da imposição de idéias e valores, embora as consequiências da punição possam não ser abusivas), supervisão do comportamento (refere-se a atitudes e comportamentos dos pais que demonstram que eles supervisionam o comportamento dos filhos buscando conhecer suas atividades ao invés de impor restrições explícitas), controle do comportamento (refere-se a atitudes e comportamentos dos pais que demonstram que eles controlam aspectos do comportamento dos adolescentes através da colocação clara de limites e imposição de regras), cobrança de responsabilidade (refere-se a atitudes e comportamentos dos pais que buscam fazer com que o adolescente se conforme às regras sociais e tenha responsabilidade por aquilo que faz), consistência (refere-se a atitudes e comportamentos dos pais que demonstram que eles são consistentes no modo de tratar os filhos, ou seja, que indicam uma certa previsibilidade nas ações e reações dos pais), aproximação afetiva (refere-se a atitudes e comportamentos dos pais que demonstram interesse dos mesmos para com o bem-estar dos adolescentes, manifestações explícitas de afeto e sensibilidade às necessidades dos jovens), apoio emocional (refere-se a atitudes e comportamentos dos pais que demonstram disponibilidade para com os filhos, fazendo-os sentirem-se seguros emocionalmente), incentivo ao desempenho (refere-se a atitudes e comportamentos dos pais que funcionam como estímulos para que o adolescente progrida nas atividades em que se envolve e desenvolva habilidades), comunicação bidirecional (refere-se a atitudes e comportamentos dos pais que demonstram disponibilidade para dialogar com os filhos, buscando compreender e aceitar opiniões divergentes), estímulo à autonomia (refere-se a atitudes e comportamentos dos pais que visam facilitar a conquista de independência psicológica dos adolescentes no âmbito das idéias e da tomada de decisões), confiança (refere-se à segurança que os pais demonstram ter no adolescente, no sentido de que o comportamento do jovem estará de acordo com suas expectativas) e intrusividade (refere-se a atitudes e comportamentos dos pais que demonstram falta de respeito à individualidade do jovem e intromissão em aspectos de sua privacidade). Note-se que as categorias de controle punitivo até cobrança de responsabilidade estão relacionadas, teoricamente, à grande dimensão de exigência; as categorias consistência até confiança correspondem à responsividade; e a categoria intrusividade é um acréscimo.

Como procedimento final nesta fase de elaboração do instrumento, os 62 itens foram submetidos à avaliação de três juízes que classificaram os itens conforme as definições de cada uma das 12 categorias (que foram fornecidas previamente aos juízes). Os itens que não foram classificados corretamente por dois ou mais juízes tiveram o seu conteúdo revisado pelos pesquisadores e foram eventualmente modificados.

\section{Estudo-Piloto}

A primeira versão do instrumento com 62 itens foi aplicada a uma amostra-piloto de 77 adolescentes (média de idade 16,8 anos), sendo 52,6\% mulheres, estudantes de terceiro ano do ensino médio de uma escola pública (onde posteriormente também foram coletados dados do estudo principal). Cada item foi respondido pelos adolescentes em relação a pai e mãe separadamente, utilizando uma escala de resposta tipo Likert com 5 pontos, com definições-âncora nos extremos da escala ( 1 =quase nunca ou bem pouco e $5=$ geralmente ou bastante) 
Estes itens foram analisados quanto à distribuição das respostas e suas correlações com os demais itens dentro de cada categoria de prática parental, uma vez que se supõe que itens de uma mesma categoria estejam medindo uma mesma dimensão de prática e, por isso, devam apresentar correlações elevadas entre si. Além disso, foram realizadas análises de consistência interna (alpha de Cronbach) para o conjunto de itens de cada categoria, com o intuito de verificar a fidedignidade de cada escala e identificar itens problemáticos. Os resultados do estudo piloto indicaram necessidade de modificações em alguns itens, que não se correlacionaram conforme o esperado. A escala de "consistência", especialmente, apresentou baixa fidedignidade e problemas em vários itens, sendo substancialmente reformulada. As demais escalas mostraram índices de fidedignidade aceitáveis para um estudo-piloto exploratório (na faixa de 0,60 a 0,80), levando-se em consideração ainda o pequeno número de itens em cada escala (em média 4 ou 5 ). Após as reformulações sugeridas pelos resultados do estudo-piloto, a versão final da escala começou a ser aplicada na amostra definitiva.

\section{Estudo Principal}

\section{Participantes}

Foram coletados dados junto a 409 adolescentes, alunos de duas escolas públicas de ensino médio de uma cidade do interior do Rio Grande do Sul (13,4\% do primeiro ano, $20,3 \%$ do segundo e $66,3 \%$ do terceiro). Destes participantes, $57,9 \%$ eram mulheres, e a média de idade do grupo foi de 16,52 anos $(d p=1,12)$. A renda familiar indicada pelos participantes foi classificada em faixas: até 1000 reais $(38,4 \%)$, de 1001 a 2000 reais $(35,3 \%)$, de 2001 a 3000 reais $(15,7 \%)$ e acima de 3000 reais $(10,6 \%)$. A escolha das escolas foi feita por conveniência, considerando-se que tratava-se de instituições tradicionais e que atendem a alunos provenientes de diversas regiões da cidade.

\section{Instrumento}

Foram utilizados inicialmente os 62 itens derivados do estudo-piloto para avaliar as práticas educativas parentais. Cada item foi respondido pelos adolescentes separadamente em relação a pai e mãe, em um sistema de resposta de 5 pontos ( $1=$ quase nunca ou bem pouco e $5=$ geralmente ou bastante). Além disso, foram ainda avaliados alguns indicadores de desenvolvimento psicossocial na adolescência para serem posteriormente correlacionados com as práticas parentais: auto-estima (através da Escala de auto-estima de Rosenberg, adaptada ao português por Hutz, 2000; alpha de Cronbach neste estudo $=0,83$ ), auto-eficácia (através da Escala de auto-eficácia percebida de Schwarzer, adaptada ao português por Teixeira e Dias, 2005; alpha de Cronbach neste estudo $=0,80$ ), abertura emocional (através de um conjunto de 4 itens elaborado para esta pesquisa, alpha de Cronbach $=0,65$ ) e comportamento anti-social (através de um conjunto de 5 itens criados para este estudo; alpha de Cronbach neste estudo $=0,55$ ). Os itens utilizados para avaliar abertura emocional foram: "Tenho dificuldade para estabelecer relações afetivas" (sentido revertido), "Tenho medo de dizer o que sinto" (revertido), "Eu tenho facilidade para demonstrar meus sentimentos" e "Para mim é difícil falar sobre o que sinto" (revertido). Por sua vez, os itens relacionados a comportamento anti-social foram: "Me envolvo em brigas com facilidade", "Costumo respeitar o patrimônio da escola ou de outros lugares públicos" (revertido), "Costumo carregar algum tipo de arma comigo", e mais dois itens relacionados ao uso de álcool e drogas.

\section{Procedimentos}

A aplicação do instrumento foi feita coletivamente em salas de aula, após esclarecimentos sobre a natureza da pesquisa e a obtenção do consentimento dos estudantes e seus pais.

\section{Análise dos Dados}

Os dados foram organizados em uma planilha e analisados utilizando-se um programa estatístico (SPSS). Inicialmente, foram feitas análises relativas à estrutura do instrumento e sua consistência interna. Posteriormente, foram calculadas as médias das variáveis de interesse e realizadas comparações (entre os sexos e entre as respostas dadas em relação a pais e mães). Por fim, o padrão de relação entre as variáveis foi investigado através de análises de correlação.

\section{Resultados}

\section{Análise de Itens e da Estrutura Componencial do Instrumento}

Análises preliminares de consistência interna (a partir das pressuposições teóricas) sugeriram a exclusão das dimensões consistência e confiança do conjunto de itens, devido à sua baixa fidedignidade. Assim, foram analisados os 51 itens referentes às dez dimensões de práticas educativas parentais restantes, a partir dos dados válidos de 298 adolescentes. Os escores de pais e mães foram combinados para a realização dessas análises (foi computada a média dos escores relativos a pai e mãe).

O conjunto de itens foi submetido a uma análise de componentes principais com o intuito de verificar se seria possível a identificação das dimensões propostas na solução componencial. Os resultados da primeira análise indicaram a necessidade de se excluir mais itens que não se mostraram úteis para a interpretação dos componentes (devido a baixas cargas componenciais - menores do que 0,30-ou cargas elevadas em mais de um fator).

Após sucessivas análises de componentes principais chegou-se a uma solução interpretável de 6 componentes para um conjunto de 27 itens (número de componentes sugerido pelo critério do eigenvalue maior do que 1), onde cada item apresentou carga superior a 0,35 em apenas um componente (foi utilizada rotação oblíqua; índice $\mathrm{KMO}=0,87$; teste de Bartlett $\operatorname{com} p<0,001)$. Ao todo, os seis componentes explicaram $58,8 \%$ da variância total (do primeiro ao sex to componente, os percentuais explicados foram: $24,3 \%, 15,3 \%$, 
$5,5 \%, 5,2 \%, 4,6 \%$ e $3,9 \%)$. A Tabela 1 apresenta os resultados desta análise, assim como a consistência interna das escalas (alpha de Cronbach). A análise do conteúdo dos itens com cargas fatoriais mais elevadas em cada componente permitiu a interpretação destes seis componentes: apoio emocional, controle punitivo, incentivo à autonomia, intrusividade, supervisão do comportamento e cobrança de responsabilidade. Análises parciais considerando-se as respostas dadas em relação a pais e mães separadamente também foram realizadas, com resultados muito similares, motivo pelo qual não são aqui apresentadas (os índices de consistência interna obtidos com as escalas de pais e mães variaram de 0,65 a 0,90$)$.

\section{Estatísticas Descritivas e Análises Correlacionais}

A Tabela 2 traz as médias e os desvios-padrão observados com esta amostra, considerando-se os escores relativos a pais e mães separadamente e também os escores combinados, por sexo. Testes t para grupos independentes mostraram diferenças significativas entre os $\operatorname{sexos}(p<0,001)$ apenas em supervisão do comportamento por parte das mães e combinado, sendo que as mulheres obtiveram escores mais altos nessas variáveis (ou seja, as mulheres perceberam suas mães isoladamente e a dupla parental combinada como mais "supervisoras" do que os homens). Já as diferenças percebidas em relação às práticas parentais de pais e mães foram analisadas através de testes $\mathrm{t}$ para grupos dependentes

Tabela 1

Resultados da Análise de Componentes Principais (Cargas Componenciais)

\begin{tabular}{|c|c|}
\hline Componentes / Itens / Alpha & Carga \\
\hline \multicolumn{2}{|l|}{ COMPONENTE I: APOIO EMOCIONAL (alpha=0,89) } \\
\hline Me incentiva a dar o melhor de mim em tudo o que eu faça. & 0,49 \\
\hline Me elogia quando eu faço uma tarefa bem feita. & 0,62 \\
\hline Demonstra carinho para comigo. & 0,81 \\
\hline Mostra interesse pelas coisas que eu faço. & 0,71 \\
\hline Encontra um tempo para estar comigo e fazermos juntos algo agradável. & 0,74 \\
\hline Procura conversar comigo. & 0,70 \\
\hline Me dá apoio emocional quando eu necessito. & 0,77 \\
\hline \multicolumn{2}{|l|}{ COMPONENTE II: CONTROLE PUNITIVO (alpha=0,73) } \\
\hline Me proíbe de fazer algo que gosto quando eu faço alguma coisa errada. & 0,72 \\
\hline Me pune de algum modo se desobedeço uma orientação sua. & 0,82 \\
\hline Impõe regras para o meu comportamento independente de minhas opiniões. & 0,37 \\
\hline Me aplica algum castigo se não concorda com o meu comportamento. & 0,81 \\
\hline \multicolumn{2}{|l|}{ COMPONENTE III: INCENTIVO À AUTONOMIA (alpha=0,76) } \\
\hline Me incentiva a agir de modo independente. & 0,74 \\
\hline Deixa eu ter minhas próprias experiências e aprender por mim mesmo(a). & 0,59 \\
\hline Me estimula para que eu tome decisões por conta própria. & 0,75 \\
\hline Me incentiva a que eu tenha minhas próprias opiniões sobre as coisas. & 0,54 \\
\hline \multicolumn{2}{|l|}{ COMPONENTE IV: INTRUSIVIDADE (alpha=0,67) } \\
\hline Mexe nas minhas coisas sem pedir permissão. & 0,70 \\
\hline Faz brincadeiras sobre assuntos meus de um jeito que eu não gosto. & 0,65 \\
\hline Dá palpite em tudo que eu faço. & 0,62 \\
\hline Se intromete em assuntos meus mesmo quando eu não peço. & 0,71 \\
\hline \multicolumn{2}{|l|}{ COMPONENTE V: SUPERVISÃO DO COMPORTAMENTO (alpha=0,77) } \\
\hline Só permite que eu saia de casa se souber aonde vou. & $-0,84$ \\
\hline Procura verificar por onde eu ando caso não saiba. & $-0,68$ \\
\hline Procura saber aonde vou quando saio de casa. & $-0,77$ \\
\hline Tem a última palavra sobre o que eu posso ou não fazer. & $-0,65$ \\
\hline \multicolumn{2}{|l|}{ COMPONENTE VI: COBRANÇA DE RESPONSABILIDADE (alpha=0,70) } \\
\hline Exige que eu vá bem na escola. & $-0,75$ \\
\hline Verifica se estou correspondendo às expectativas nos estudos. & $-0,69$ \\
\hline Me cobra que eu seja organizado(a) com as minhas coisas. & $-0,69$ \\
\hline Me cobra se eu não levo a sério meus compromissos. & $-0,39$ \\
\hline
\end{tabular}

Nota. Todos os itens listados apresentaram cargas superiores a 0,35 em apenas um componente. 
(medidas repetidas). Os resultados dessas análises indicaram diferenças estatisticamente significativas tanto na percepção dos homens quanto das mulheres em supervisão do comportamento, cobrança de responsabilidade, apoio emocional e intrusividade $(p=0,014$ para homens em supervisão; nas demais análises $p<0,001)$. Para a variável estímulo à autonomia, observou-se diferença significativa apenas entre as mulheres $(p<0,001)$. Em todos os casos, as pontuações mais altas foram atribuídas às práticas parentais das mães.

Tabela 2

Médias (e Desvios-Padrão) por Subescala

\begin{tabular}{|c|c|c|}
\hline \multicolumn{3}{|c|}{ Médias (e Desvios-Padrão) por Subescala } \\
\hline & Mulheres & Homens \\
\hline \multicolumn{3}{|c|}{ 1. Controle punitivo } \\
\hline mães & $2,61(1,07)$ & $2,56(1,04)$ \\
\hline pais & $2,51(1,10)$ & $2,57(1,04)$ \\
\hline combinado & $5,09(1,92)$ & $5,15(1,97)$ \\
\hline \multicolumn{3}{|c|}{ 2. Supervisão do comportamento } \\
\hline mães & $4,22(0,88)$ & $3,76(1,04)$ \\
\hline pais & $3,82(1,17)$ & $3,63(1,12)$ \\
\hline combinado & $8,04(1,71)$ & $7,41(2,01)$ \\
\hline \multicolumn{3}{|c|}{ 3. Cobrança de responsabilidade } \\
\hline mães & $4,22(0,80)$ & $4,15(0,86)$ \\
\hline pais & $3,81(1,02)$ & $3,87(1,00)$ \\
\hline combinado & $8,03(1,61)$ & $8,02(1,68)$ \\
\hline \multicolumn{3}{|c|}{ 4. Intrusividade } \\
\hline mães & $2,38(1,06)$ & $2,30(0,99)$ \\
\hline pais & $1,96(0,88)$ & $1,97(0,87)$ \\
\hline combinado & $4,31(1,59)$ & $4,28(1,67)$ \\
\hline \multicolumn{3}{|c|}{ 5. Apoio emocional } \\
\hline mães & $4,21(0,88)$ & $4,13(0,79)$ \\
\hline pais & $3,60(1,21)$ & $3,79(0,99)$ \\
\hline combinado & $7,82(1,81)$ & $7,91(1,62)$ \\
\hline \multicolumn{3}{|c|}{ 6. Estímulo à autonomia } \\
\hline mães & $3,96(0,96)$ & $3,86(0,85)$ \\
\hline pais & $3,61(1,12)$ & $3,81(0,96)$ \\
\hline combinado & $7,57(1,83)$ & $7,63(1,65)$ \\
\hline
\end{tabular}

As escalas obtidas a partir da análise de componentes principais foram ainda correlacionadas, exploratoriamente, com quatro variáveis tipicamente estudadas em pesquisas sobre práticas ou estilos parentais e desenvolvimento psicossocial na adolescência: auto-estima, auto-eficácia, abertura emocional e comportamento anti-social (por exemplo, Beyers \& Goossens, 1999; Lamborn et al., 1991). A Tabela 3 exibe os resultados das correlações, assim como as correlações entre as subescalas (considerando os escores combinados de pais e mães).

Como se pode observar, controle punitivo e instrusividade mostraram-se negativamente correlacionadas com auto-estima, auto-eficácia e abertura emocional. Ainda, a intrusividade correlacionou-se positivamente com comportamento anti-social. A supervisão do comportamento e o apoio emocional, por sua vez, correlacionaram-se negativamente com comportamento anti-social. Por fim, a cobrança de responsabilidade, o apoio emocional e o estímulo à autonomia tiveram correlação positiva com auto-estima, autoeficácia e abertura emocional.

\section{Discussão}

Os resultados da análise fatorial indicam que é possível distinguir empiricamente dimensões de práticas educativas parentais que, de algum modo, estão relacionadas às dimensões mais amplas de responsividade e exigência (além da dimensão extra de intrusividade aqui incluída), embora nem todas as dimensões propostas tenham sido identificadas. De fato, das doze dimensões propostas, apenas seis emergiram da análise. Apoio emocional, aproximação afetiva e incentivo ao desempenho tenderam a se agrupar em um fator apenas, chamado aqui de apoio emocional; supervisão e controle do comportamento também não se distinguiram empiricamente, formando um fator denominado supervisão do comportamento; comunicação bi-direcional, por sua vez, não emergiu das primeiras análises como um fator claro, e seus itens foram retirados do conjunto. Assim, resultou um modelo fatorial de seis dimensões: apoio emocional, controle punitivo, incentivo à autonomia, intrusividade, supervisão do comportamento e cobrança de responsabilidade. Os índices de consistência interna obtidos para cada uma das escalas podem ser considerados satisfatórios, dado o pequeno número de itens em cada escala.

Tabela 3

Correlações entre as Dimensões de Práticas Educativas Parentais e demais Variáveis

\begin{tabular}{|c|c|c|c|c|c|c|c|c|c|}
\hline & (1) & $(2)$ & (3) & $(4)$ & $(5)$ & (6) & $(7)$ & (8) & (9) \\
\hline 1. Controle punitivo & - & & & & & & & & \\
\hline 2. Supervisão do comportamento & $0,40^{*}$ & - & & & & & & & \\
\hline 3. Cobrança de responsabilidade & $0,30 *$ & $0,46^{*}$ & - & & & & & & \\
\hline 4. Intrusividade & 0,37 * & 0,23 * & $0,14^{*}$ & - & & & & & \\
\hline 5. Apoio emocional & $-0,05$ & $0,29^{*}$ & $0,48^{*}$ & $-0,19^{*}$ & - & & & & \\
\hline 6. Estímulo à autonomia & $-0,11^{*}$ & 0,09 & $0,26 *$ & $-0,27^{*}$ & $0,59^{*}$ & - & & & \\
\hline 7. Auto-estima & $-0,16^{*}$ & 0,03 & $0,10^{*}$ & $-0,10^{*}$ & $0,24^{*}$ & $0,21^{*}$ & - & & \\
\hline 8. Auto-eficácia & $-0,10 *$ & 0,03 & 0,16 * & $-0,11 *$ & $0,26 *$ & $0,26 *$ & $0,52^{*}$ & - & \\
\hline 9. Abertura emocional & $-0,11 *$ & 0,01 & $0,13 *$ & $-0,12$ * & $0,18 *$ & $0,12 *$ & $0,35 *$ & $0,34^{*}$ & - \\
\hline 10. Comportamento anti-social & 0,06 & $-0,19 *$ & $-0,09$ & $0,15^{*}$ & $-0,22 *$ & $-0,08$ & $-0,13^{*}$ & $-0,15^{*}$ & $-0,03$ \\
\hline
\end{tabular}

Nota. ${ }^{*} p<0,05$ (correlações de Pearson) 
Estes resultados sugerem que, ao se estudar o impacto das práticas parentais sobre o comportamento adolescente, devem ser consideradas as especificidades de cada prática. Ou seja, ainda que possam estar correlacionadas entre si as diversas dimensões, elas se referem a diferentes comportamentos que os pais têm para com os filhos, e o impacto destas práticas específicas pode ser diferente para o desenvolvimento psicossocial dos adolescentes. De fato, embora muitas pesquisas combinem as dimensões de práticas formando estilos ou mesmo dimensões amplas como responsividade ou exigência, existem estudos que sugerem diferenças de efeitos para práticas mais específicas (por exemplo, Claes et al., 2003; Kenny, \& Gallagher, 2002; Meesters, \& Muris, 2004; Sim, 2003; Vazsonyi, Hibbert, \& Snider, 2003).

Os resultados das análises correlacionais estão de acordo com essa hipótese. O controle punitivo e a intrusividade, por exemplo, mostraram-se negativamente correlacionadas com auto-estima, auto-eficácia e abertura emocional, sugerindo que são variáveis bastante associadas, ainda que distintas. No entanto, a supervisão do comportamento, que também está associada à idéia de exigência parental, não apresentou esse tipo de correlação. Ou seja, a supervisão, embora represente um controle dos pais sobre o comportamento dos filhos, não parece prejudicar nem favorecer o desenvolvimento da estima, da eficácia e da abertura emocional dos mesmos (ao menos do modo como foi avaliada neste estudo). Este efeito diferencial se deve, possivelmente, a uma certa ambiguiidade presente na idéia de supervisão do comportamento, que pode ser entendido como um componente específico de um construto mais amplo chamado de monitoração. Segundo Kerr, Stattin e Trost (1999), níveis altos de monitoração do comportamento podem indicar alto grau de confiança parental relacionada à previsibilidade do comportamento de seus filhos. Dessa forma, a monitoração pode se efetivar não através de comportamentos ativos dos pais na tentativa de buscar saber o que o filho faz ou deixa de fazer, mas através da própria revelação espontânea, por parte dos filhos, de seus comportamentos. Assim, os pais monitoram seus filhos através das informações que estes lhes passam, e só intervêem de um modo mais ativo caso essa informação não seja suficiente. Essa regulação bi-direcional de comportamentos de pais (através da monitoração) e filhos (através da auto-revelação) não assume, dessa forma, características intrusivas que podem afetar a auto-estima e a autoeficácia dos jovens. Note-se que, nessa concepção de monitoração, está implícita a presença tanto de supervisão quanto de uma certa aceitação emocional por parte dos pais, um aspecto que a escala utilizada neste estudo não contemplou explicitamente em seu conteúdo. Contudo, a correlação positiva observada entre supervisão do comportamento e apoio emocional sugere que a supervisão parental é percebida pelos adolescentes como algo positivo, e não intrusivo.

Por outro lado, conforme mencionado anteriormente, os dados sugerem que comportamentos punitivos por parte dos pais, bem como a intrusividade, tendem a estar associados a efeitos negativos no desenvolvimento dos filhos, afetando negativamente a auto-estima, a auto-eficácia e a abertura emocional (embora deva-se considerar que a magnitude dos efeitos observados foi pequena). Resultados semelhantes foram obtidos por Xia e Qian (2001), que observaram associações positivas de práticas parentais com características coercitivas (uso de penalidades, proteção excessiva, rejeição e negação) com sintomas psicossomáticos e negativas com diversos indicadores de saúde mental geral. De fato, Cecconello, De Antoni e Koller (2003) ressaltam que um controle excessivo do comportamento dos filhos pode inibir o desenvolvimento de habilidades importantes, com possíveis consequiências para a auto-estima e a autonomia. Os autores salientam, ainda, que a utilização de punição física como prática disciplinar pode gerar desequilíbrio nos filhos, além de prejudicar a relação de afeto e reciprocidade com os genitores. Assim, é possível que o uso de punição e a intromissão parental façam com que os adolescentes sintam-se amedrontados de exporem seus sentimentos e também não se lancem em atividades exploratórias saudáveis que propiciariam o desenvolvimento de sua auto-eficácia e conseqüentemente de sua auto-estima. Esse efeito poderia ser gerado em virtude de um possível afastamento dos filhos em relação aos pais provocado pelos comportamentos punitivos e intrusivos, prejudicando a construção de uma relação de apego apropriada para o desenvolvimento de competências relacionais e pessoais que permitam segurança no momento em que o adolescente coloca-se de maneira mais autônoma em atividades exploratórias (Kenny \& Gallagher, 2002). É importante lembrar, contudo, que o controle punitivo, do modo como foi operacionalizado nesta pesquisa, não implica necessariamente o uso de punições abusivas por parte dos pais (o que caracterizaria mais a coerção). Ainda, é preciso considerar que os efeitos negativos do controle punitivo aqui observados podem, na verdade, depender do padrão global de práticas educativas, não se podendo concluir que o uso de punição, por si só, seja determinante de problemas.

Já a variável comportamento anti-social mostrou-se associada negativamente com supervisão do comportamento e com apoio emocional dos pais. Este resultado está de acordo com outros estudos que observaram relações negativas entre indicadores de comportamento anti-social ou delinqüente com variáveis como proximidade, apoio, sensibilidade e monitoração parental (Griffin, Botvin, Scheier, Diaz, \& Miller, 2000; Juang \& Silbereisen, 1999; Vazsonyi, Hibbert \& Snider, 2003). Porém, não foi observada correlação significativa entre comportamento anti-social e controle punitivo. Poder-se-ia pensar que um controle mais punitivo por parte dos pais (mas não propriamente coercitivo) favorecesse o seguimento de regras e normas e assim se correlacionasse negativamente com comportamento antisocial. Contudo, também há indicações na literatura (Gomide, 2003) de que práticas coercitivas (o que inclui punições com conseqüências abusivas) podem se associar positivamente com comportamento anti-social. Uma vez que a escala aqui utilizada não especificou a gravidade das consequiências da punição parental, a ausência de correlação 
pode ter sido resultado de tal inespecificidade pois, para alguns adolescentes, o controle punitivo (conforme operacionalizado) pode ter características mais coercitivas, enquanto para outros não. Tais interpretações relativas ao comportamento anti-social, contudo, devem ser tomadas apenas como possibilidades que merecem novas investigações, até mesmo porque a medida usada neste estudo foi bastante limitada.

Por outro lado, cobrança de responsabilidade, estímulo à autonomia e apoio emocional mostraram um padrão de correlações positivas com auto-estima, auto-eficácia e abertura emocional muito semelhante, o que indica que estas variáveis estão associadas. Este resultado confere alguma validade às escalas construídas, pois sabe-se que as dimensões relacionadas com a responsividade têm repercussões importantes no âmbito do desenvolvimento das competências interpessoais e na valorização de si-mesmo (Steinberg, 2001).

Por fim, as comparações entre homens e mulheres e entre os escores atribuídos a pais e mães mostraram resultados similares aos de outras pesquisas. As mulheres perceberam uma maior supervisão por parte de mães e da dupla parental combinada, o que está de acordo com os estudos de Costa, Teixeira e Gomes (2000) e Weber et al. (2004), que verificaram níveis mais altos de exigência percebidos por parte das mulheres (note-se que a monitoração pode ser entendida como um aspecto da dimensão mais ampla de exigência). Por sua vez, nas variáveis relacionadas a responsividade (apoio emocional e estímulo à autonomia) não foram detectadas diferenças significativas entre os sexos, novamente concordando com os achados dos estudos citados anteriormente no que diz respeito aos escores combinados de pais e mães. Já na comparação dos escores atribuídos a mães e pais, o padrão geral observado também foi o mesmo das pesquisas mencionadas, ou seja, o nível percebido de práticas parentais maternas foi superior ao de práticas paternas, o que indica a maior saliência do papel materno na educação dos filhos, ao menos na percepção dos adolescentes.

Em síntese, este estudo atingiu o seu objetivo principal que foi a elaboração de um instrumento para avaliar dimensões de práticas educativas parentais na adolescência, propondo e identificando empiricamente escalas para avaliar outras dimensões além de responsividade e exigência, já tradicionalmente apontadas na literatura. As escalas construídas mostraram-se empiricamente distintas através da análise de componentes principais e seus índices de fidedignidade foram aceitáveis para pesquisa. Por fim, as correlações entre as práticas parentais e as variáveis de desenvolvimento psicossocial obtidas foram compatíveis com o que é relatado na literatura, indicando que as práticas educativas parentais são importantes para o desenvolvimento psicossocial dos adolescentes e sugerindo, ainda, a validade das medidas utilizadas. Sugere-se que futuros estudos busquem investigar outros possíveis efeitos específicos que essas subdimensões possam ter sobre aspectos do desenvolvimento psicossocial de adolescentes, além de explorar outras dimensões de práticas educativas não abordadas nesta pesquisa.

\section{Referências}

Bardagi, M. P. (2002). Os estilos parentais e sua relação com a indecisão profissional, ansiedade e depressão dos filhos adolescentes. Dissertação de Mestrado não-publicada, Instituto de Psicologia, Universidade Federal do Rio Grande do Sul, Porto Alegre, RS.

Benetti, S. P. da C., \& Balbinotti, M. A. A. (2003). Elaboração e estudo de propriedades psicométricas do Inventário de Práticas Parentais. Psico-USF, 8, 103-113.

Beyers, W., \& Goossens, L. (1999). Emotional autonomy, psychosocial adjustment and parenting: Interactions, moderating and mediating effects. Journal of Adolescence, 22, 753-769.

Cecconello, A. M., De Antoni, C., \& Koller, S. H. (2003). Práticas educativas, estilos parentais e abuso físico no contexto familiar. Psicologia em Estudo, 8, 45-54.

Claes, M., Lacourse, E., Bouchard, C., \& Perucchini, P. (2003). Parental practices in late adolescence, a comparison of three countries: Canada, France and Italy. Journal of Adolescence, 26, 387-399.

Costa, F. T. da, Teixeira, M. A. P., \& Gomes, W. B. (1998). Estilo parental na percepção de adolescentes [Resumo]. In Universidade Federal do Rio Grande do Sul (Ed.), Resumos do X Salão de Iniciação Científica (pp. 425). Porto Alegre, RS: Editora da Universidade Federal do Rio Grande do Sul.

Costa, F. T. da, Teixeira, M. A. P., \& Gomes, W. B. (2000). Responsividade e exigências: Duas escalas para avaliar estilos parentais. Psicologia: Reflexão e Crítica, 13, 465-473.

Darling, N., \& Steinberg, L. (1993). Parenting style as context: An integrative model. Psychological Bulletin, 113, 487-493.

Gomide, P. I. C. (2003). Estilos parentais e comportamento antisocial. In A. Del Prette \& Z. A. P. Del Prette (Eds.), Habilidades sociais, desenvolvimento e aprendizagem (pp. 21-60). Campinas, SP: Alínea.

Griffin, K. W., Botvin, G. J., Scheier, L. M., Diaz, T. \& Miller, N. L. (2000). Parenting practices as predictors of substance use, delinquency, and aggression among urban minority youth: Moderating effects of family structure and gender. Psychology of Addictive Behaviors, 14, 174-184.

Hutz, C. S. (2000). Adaptação brasileira da escala de auto-estima de Rosenberg. Manuscrito não-publicado, Curso de Pós-Graduação em Psicologia do Desenvolvimento, Universidade Federal do Rio Grande do Sul, Porto Alegre, RS.

Juang, L. P., \& Silbereisen R. K. (1999). Supportive parenting and adolescent adjustment across time in former East and West Germany. Journal of Adolescence, 22, 719-736.

Juang, L. P., \& Silbereisen, R. K. (2002). The relationship between adolescent academic capability beliefs, parenting and school grades. Journal of Adolescence, 25, 3-18.

Kenny, M. E., \& Gallagher, L. A. (2002). Instrumental and social/ relational correlates of perceived maternal and paternal attachment in adolescence. Journal of Adolescence, 25, 203-2 19.

Kerr, M., Stattin, H., \& Trost K. (1999). To know you is to trust you: Parents trust is rooted in child disclosure of information. Journal of Adolescence, 22, 737 - 752. 
Laible, D. J., \& Carlo, G. (2004). The differential relations of maternal and paternal support and control to adolescent social competence, self-worth, and sympathy. Journal of Adolescent Research, 19, 759-782.

Lamborn, S. D., Mounts, N. S., Steinberg, L., \& Dornbusch, S. M. (1991). Patterns of competence and adjustment among adolescents from authoritative, authoritarian, indulgent, and neglectful families. Child Development, 62, 1049-1065.

Maccoby, E., \& Martin, J. (1983). Socialization in the context of the family: Parent-child interaction. In E. M. Hetherington (Ed.), P. H. Mussen (Series Ed.), Handbook of child Psychology: Vol. 4. Socialization, personality, and social development (pp. 1101). New York: Wiley.

Meesters, C., \& Muris, P. (2004). Perceived parental rearing behaviors and coping in young adolescents. Personality and Individual Differences, 37, 513-522.

Pacheco, J. T., Teixeira, M. A. P., \& Gomes, W. B. (1999). Estilos parentais e habilidades sociais na adolescência. Psicologia: Teoria e Pesquisa, 15, 117-126.

Reppold, C. T. (2001). Estilo parental percebido e adaptação psicológica de adolescentes adotados. Dissertação de Mestrado nãopublicada, Instituto de Psicologia, Universidade Federal do Rio Grande do Sul, Porto Alegre, RS.

Shucksmith, J., Hendry, L. B. \& Glendinning, A. (1995). Models of parenting: Implications for adolescent well-being within different types of family contexts. Journal of Adolescence, 18 , 253-270.

Sim, T. N. (2003). The father-adolescent relationship in the context of the mother-adolescent relationship: Exploring moderating linkages in a late-adolescent sample in Singapore. Journal of Adolescent Research, 18, 383-404.
Steinberg, L. (2001). We know some things: Parent-adolescent relationships in retrospect and prospect. Journal of Research on Adolescence, 11, 1-19.

Teixeira, M. A. P., Bardagi, M. P., \& Gomes, W. B. (2004). Refinamento de um instrumento para avaliar responsividade e exigência parental percebidas na adolescência. Avaliação Psicológica, 3, 1-12

Teixeira, M. A. P., \& Dias, A. C. G. (2005). Propriedades psicométricas da versão traduzida para o português da Escala de Auto-eficácia Geral Percebida de Ralph Schwarzer [Resumo]. In Instituto Brasileiro de Avaliação Psicológica (Ed.), Anais do II Congresso Brasileiro de Avaliação Psicológica [CDROM]. Gramado, RS: IBAP.

Vazsonyi, A. T., Hibbert, J. R., \& Snider, B. (2003). Exotic enterprise no more? Adolescent reports of family and parenting processes from youth in four countries. Journal of Research on Adolescence, 13, 129-160.

Weber, L. N. D., Prado, P. M., Viezzer A. P., \& Brandenburg, O. J. (2004). Identificação de estilos parentais: o ponto de vista dos pais e dos filhos. Psicologia: Reflexão e Crítica, 17, 323-331.

Xia, G., \& Qian, M. (2001). The relationship of parenting style to self-reported mental health among two subcultures of Chinese. Journal of Adolescence, 24, 251-260.
Recebido: 09/09/2005

$1^{a}$ revisão: $2 / 12 / 2005$

$2^{a}$ revisão: $30 / 01 / 2006$

Aceite final: 10/02/2006 